\title{
DETERMINAÇÃO DE PARÂMETROS FÍSICOS DE SISTEMAS BINÁRIOS ECLIPSANTES DE CURTO PERÍODO
}

\author{
Braian Barbosa de Oliveira'; ${ }^{1}$ Marildo Geraldête Pereira ${ }^{2}$; \\ 1. Bolsista FAPESB/UEFS, Graduando em Física, Universidade Estadual de Feira de Santana: \\ E-mail: braiandeoliveira@gmail.com \\ 2. Orientador, Departamento de Física, Universidade Estadual de Feira de Santana. \\ E-mail: marildogp@gmail.com
}

PALAVRAS-CHAVE: Sistema Binário, Fotometria, PHOEBE

\section{INTRODUÇÃO}

Dentro do grupo das estrelas variáveis, um tipo específico de variáveis, as binárias eclipsantes, valem ser destacadas por alguns motivos principais. Primeiramente, por serem extremamente numerosas, de acordo com Helmut (1983) quase 60\% das estrelas em nossa Galáxia estão em sistemas estelares binários ou em sistemas múltiplos. Outro motivo que torna os sistemas binários imensamente importantes para os astrônomos é por permitirem a determinação de parâmetros fundamentais para a construção do conhecimento sobre as propriedades estelares, parâmetros como a massa, que pode ser determinada a partir da Lei de Kepler, uma vez que seja conhecido o período orbital do sistema. O estudo da evolução de estrelas binárias também pode contribuir para explicar a vasta diversidade de fenômenos e objetos que vemos no universo, como variáveis cataclísmicas, binárias de Raio-X e alguns tipos de supernovas. Desta forma, pode-se afirmar que com o estudo desse tipo de sistema, estaremos contribuindo com o entendimento da estrutura e da evolução das estrelas, e de forma geral, contribuindo com o desenvolvimento do conhecimento sobre os processos físicos de evolução do universo como um todo.

Sob esse cenário, o trabalho desenvolveu uma análise sob os sistemas binários eclipsantes utilizando duas abordagens distintas para determinar os parâmetros físicos dos sistemas. No caso de NSVS 14256825, fonte observada pelo nosso grupo de pesquisa, todo o processamento das imagens, tratamento e análise foi realizado sob a plataforma IRAF enquanto com KIC 10544976, o processo foi realizado com os dados públicos do satélite Kepler, e analisados com o programa PHOEBE, sistema que despertou interesse graças ao seu período orbital relativamente curto $(\sim 8.4 \mathrm{~h})$ e a sua fase evolutiva, uma binária pós-envelope comum (PCEB), que de acordo com Pyrzas et al. (2009) compõe uma pequena parcela dos sistemas binários eclipsantes conhecidos, chegando a apenas a 14 objetos. Para estudo de sistemas desta natureza se faz necessário o uso de ferramentas que permitam a modelagem e determinação de parâmetros dos mesmos, entretanto esta tarefa nem sempre é simples, pois via de regra os programas destinados a esse fim são complexos e demandam a manipulação de uma grande quantidade de parâmetros. Nesse sentido, este trabalho também teve por objetivo o desenvolvimento de uma metodologia de modelagem de sistemas binários eclipsantes utilizando o software gratuito PHOEBE (PHysics Of Eclipsing BinariEs), desenvolvido por Prša \& Zwitter (2005) baseado na geometria de Roche utilizando o código Wilson-Devinney (1971). 


\section{METODOLOGIA}

Na primeira fase do trabalho, foi feito o tratamento e redução das imagens obtidas de NSVS 14256825 pelo grupo de pesquisa no OPD (Observatório Pico dos Dias), através da plataforma IRAF. A partir desse momento, com as imagens reduzidas e a curva de luz obtida pudemos prosseguir com a análise fotométrica do sistema a partir das rotinas de modelagem através do código Wilsson\&Devinney. O qual foi utilizado juntamente com o método MCMC para a obtenção da solução para o sistema, com a obtenção dos parâmetros físicos e geométricos do mesmo. Com KIC 10544976 o trabalho se iniciou com a obtenção dos dados fotométricos de KIC 10544976, os quais foram obtidos através do banco de dados online do telescópio Kepler ${ }^{1}$. Em seguida, através da revisão bibliográfica obtivemos alguns parâmetros importantes para desenvolvimento da metodologia proposta, utilizando o artigo "An eclipsing post-common-envelope binary in the field of the Kepler mission" (ALMENARA et al., 2012). No qual os autores determinaram através de dados fotométricos, espectroscópicos e das curvas de velocidade radial, vários parâmetros físicos do sistema e das componentes. Em seguimento buscou-se inicialmente entender como esses parâmetros físicos e geométricos se relacionam com o formato das curvas de luz obtidas pelo modelo teórico previsto no software para o sistema, e como a curva sintética gerada pelo PHOEBE se comporta com o ajuste de cada parâmetro individualmente, buscando entender qual a contribuição desses parâmetros na obtenção da solução para o sistema.

Após ter compreendido a contribuição individual de cada um desses parâmetros nas curvas e no formato da superfície das estrelas, é dado início à modelagem do sistema. Partindo dos valores obtidos na literatura em busca da obtenção de uma curva sintética próxima ao observado e uma configuração geométrica que represente melhor o sistema diante do modelo previsto na literatura. Onde, após calcularmos o valor dos parâmetros desejados checávamos na guia de plotagem o quanto a curva de luz sintética calculada se aproximava da curva observada, salvando o arquivo de resultados após cada interação bem-sucedida, em seguida, os dados são atualizados e obtida uma nova curva sintética, buscando com isso, a cada passo se aproximar da melhor solução para o sistema.

\section{RESULTADOS E DISCUSSÃO}

Pudemos através da metodologia proposta para a modelagem do sistema binário NSVS 14256825, utilizando o IRAF, obter os valores para alguns parâmetros do sistema e de suas componentes. Os quais são exibidos na Tabela 1:

Tabela 1 - Parâmetros obtidos para o sistema NSVS 14256825

\begin{tabular}{lll}
\hline \multicolumn{1}{c}{ Parâmetro } & Valor Obtido & \multicolumn{1}{c}{$\begin{array}{c}\text { Valor da } \\
\text { Literatura }\end{array}$} \\
\hline Razão de Massa $\left(\mathrm{M}_{2} / \mathrm{M}_{1}\right)$ & 0.260 & 0,26 \\
Inclinação $\left(^{\circ}\right)$ & $82^{\circ} .473$ & $82^{\circ}, 5$ \\
$\mathrm{~T}_{\text {Eff Primária }}$ & $42300 \mathrm{~K}$ & $40000 \mathrm{~K}$ \\
$\mathrm{~T}_{\text {Eff Secundária }}$ & $2400 \mathrm{~K}$ & $2400 \mathrm{~K}$ \\
Potencial Primária & 4.580 & - \\
Potencial Secundária & 2.799 & - \\
& $\dagger$ Almeida, L. A. et al. 2012
\end{tabular}

\footnotetext{
${ }^{1}$ https://exoplanetarchive.ipac.caltech.edu/applications/ETSS/Kepler_index.html
} 
Os valores obtidos se aproximam muito bem dos valores da literatura, apesar do tempo de observação, e quantidade de curvas modeladas serem pequenas em comparação ao trabalho de Almeida, L. A. et al. I2012). Isso se deve ao fato de que a metodologia empregada no IRAF ser similar a metodologia utilizada pelos autores no trabalho referenciado.

Para a modelagem feita do sistema KIC-10544976 através da metodologia empregada no PHOEBE, foram obtidos os resultados que são exibidos na Tabela 2. Podemos perceber que alguns valores apresentam uma diferença acentuada quando comparados aos dados da literatura, isso acontece devido a modelagem ter sido feita apenas sob alguns parâmetros, e através apenas de dados fotométricos. Contudo a solução, com a curva sintética obtida e o perfil das superfícies equipotenciais dos sistemas coincidem com o esperado.

Tabela 2 - Apresentação dos valores obtidos no melhor ajuste para o sistema KIC-10544976

\begin{tabular}{lll}
\hline \multicolumn{1}{c}{ Parâmetro } & \multicolumn{1}{c}{ Valor Obtido } & Valor da Literatura \\
\hline Razão de Massa $(q)$ & $0,62 \pm 0,05$ & 0,63 \\
Inclinação (i) & $97^{\circ}, 9 \pm 0,2$ & $89^{\circ}, 5 \pm 1,5$ \\
TEff Primária $_{\text {TEff Secundária }}$ & $25555 \pm 453 \mathrm{~K}$ & $20470 \pm 1300 \mathrm{~K}$ \\
$\begin{array}{l}\text { Potencial na Superfície } \\
\text { da Primária }\left(\Omega_{1}\right)\end{array}$ & $4034 \pm 33 \mathrm{~K}$ & $3200 \pm 100 \mathrm{~K}$ \\
$\begin{array}{l}\text { Potencial na Superfície } \\
\text { da Secundária }\left(\Omega_{2}\right)\end{array}$ & $4,05 \pm 0,02,38$ & 74.149 \\
\hline
\end{tabular}

ALMENARA et al., $2012 \dagger$.
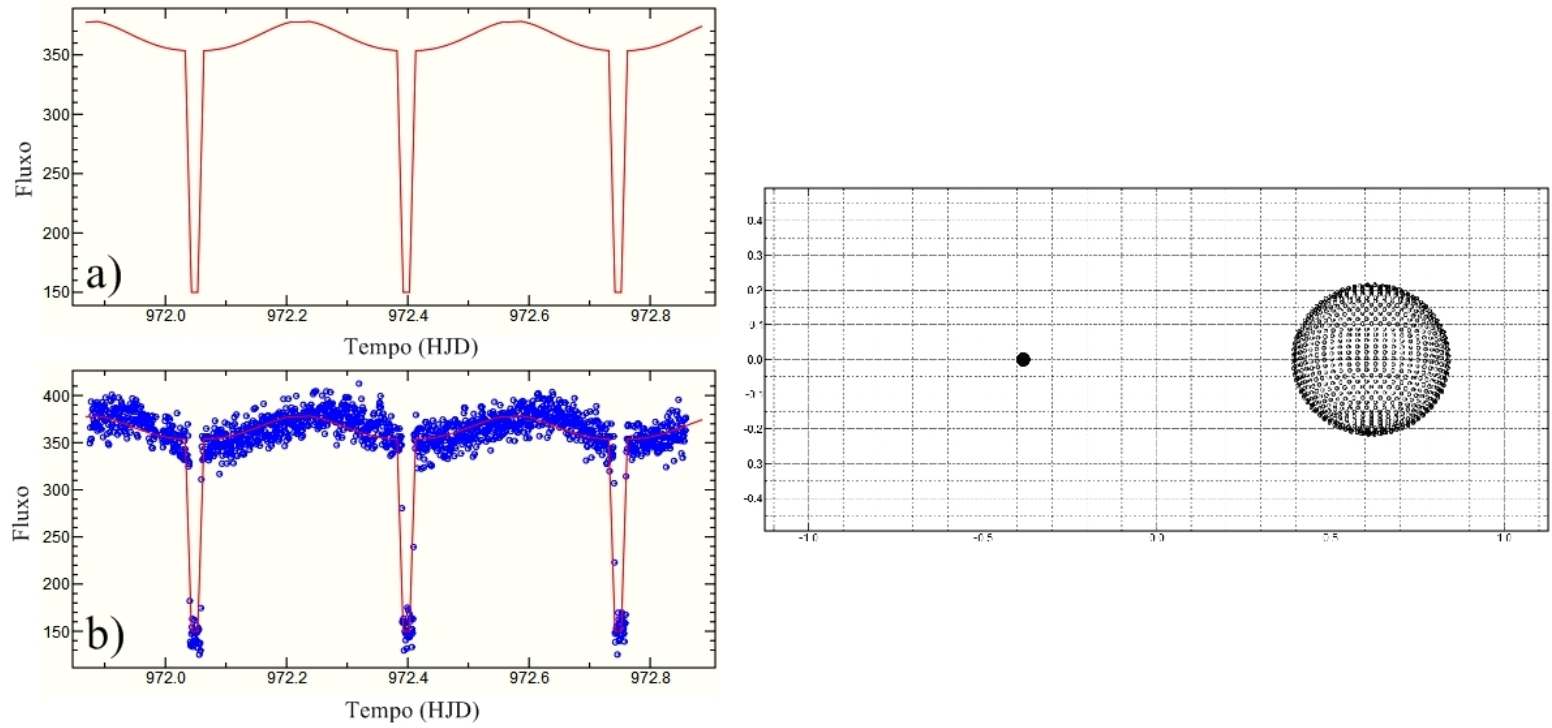

Figura 1- a) Curva sintética obtida através da modelagem. b) Curva Sintética Obtida a partir dos parâmetros do ajuste sobreposta aos valores observados, e configuração geométrica obtida do sistema à direita.

\section{CONSIDERAÇÕES FINAIS}

Este trabalho teve como proposta a determinação de parâmetros de sistemas binários eclipsantes e o desenvolvimento de uma metodologia para modelagem desses sistemas, a qual foi alcançada com o uso do IRAF e utilizando o PHOEBE. Tendo em vista que, para estudo de sistemas desta natureza se faz necessário o uso de ferramentas que permitam a modelagem e determinação de parâmetros dos mesmos, não sendo necessariamente uma tarefa simples, 
pois via de regra os programas destinados a esse fim são complexos e demandam a manipulação de uma grande quantidade de parâmetros, foi desenvolvido nesse trabalho também um guia com intuito de reforçar e guiar trabalhos futuros sobre essa temática, além de uma modelagem preliminar do sistema binário eclipsante KIC 10544976.

Contudo, vale ressaltar que a metodologia proposta e aplicada na análise de KIC 10544976 com o PHOEBE levou em conta apenas alguns parâmetros principais de sistemas estelares binários eclipsantes. Partindo apenas da análise de dados fotométricos e dos dados da literatura. $\mathrm{O}$ que evidentemente pode ter influenciado e comprometido os resultados expostos na Tabela 2. Deve ser notado também, que no PHOEBE, são cerca de 40 parâmetros que o usuário pode configurar para a obtenção de um bom resultado, parâmetros como os valores de albedo, efeitos de reflexão, brilho gravitacional e parâmetros de sincronização da órbita, que foram ignorados por falta de informações sobre o sistema.

Contudo, mesmo com tais dificuldades consideramos os resultados obtidos e os avanços na metodologia construída importantes no ponto de vista da contribuição com trabalhos futuros. Diante deste cenário, podemos considerar o PHOEBE uma interessante ferramenta para o estudo de sistemas estelares binários eclipsantes, de modo que a perspectiva da expansão do estudo sobre o funcionamento desse software a partir desse trabalho pode contribuir para o estudo e determinação de parâmetros através da modelagem de sistemas binários eclipsantes.

\section{REFERÊNCIAS}

[1] AITKEN R. G. The Binary Stars.New York: Dover, 1964, p. ix.

[2] ALMENARA, J. M. et al. An eclipsing post-common-envelope binary in the field of the Kepler mission. 2012, Mon. Not. R. Astron. Soc., 420, 3017. [3] ALMEIDA, L. A., JABLONSKI, F., TELlO, J., \& RODRIGUES, C. V. A photometric and spectroscopic study of NSVS 14256825: the second sdOB+dM eclipsing binary_2012, MNRAS, 423,

[4] ALMEIDA L. A., Atividade nas Secundáarias de Variáveis Cataclísmicas. INPE, 2009. Dissertação de Mestrado, São José dos Campos.

[5] ABT, H. A. Normal and abnormal binary frequencies. Annual review of astronomy and astrophysics, v. 21, p. 343-372, 1983.

[6] CÉSAR, J. Análise de Binárias Eclipsantes no Bojo da Galáxia com dados OGLE-II usando o código Wilson-devinney. INPE, 2012. Tese de Doutorado, São José dos Campos. Disponível em: <http://urlib.net/8JMKD3MGP7W/3DG4UD5>

[7] FANELLI M. N., Kepler: A Search for Terrestrial Planets. Kepler Data Processing Handbook . Moffett Field, CA. Abril, 2011. 108.

[8] FILHO, K. S.; SARAIVA, M. d. F. O. Astronomia e Astrofísica. 2a Edição. São Paulo: Livraria da Física, 2004.

[9] HILL G., RUCINSKI S.M.; Light Curve Modeling of Eclipsing Binary Stars. 1993, 135. [10] KOPAL, Z. The classification of close binary systems. 1955. Annalesd'Astrophysique, v. 18, p. 379-428.

[11] PYRZAS S. et al., Post-common-envelope binaries from SDSS. 2009, MNRAS, 394. [12] WILSON, R.E., \& DEVINNEY, E.J. Realization of Accurate Close Binary Light Curve: Application to MR Cygni. 1971, ApJ, 166, 605.

[13] WOOD D. B.; A Computer Program for Modeling Non-Spherical Eclipsing Binary Star Systems. 1972. Technical Report X-110-72-473. GSFC, Greenbelt, MD.

[14] ZASCHE, P. PHOEBE - step by step manual. 2016. Open European Journal on Variable Stars, vol. 176. 